Grenzrelationen 


\title{
Grenzen und Europa
}

\author{
Monika Eigmüller
}

\begin{abstract}
Kern des europäischen Integrationsprojekts ist die Verwirklichung der vier Binnenfreiheiten: freier Verkehr von Kapital, Waren, Dienstleitungen und Personen. Deren Realisierung setzt zugleich den Abbau von Binnengrenzkontrollen zwischen den Mitgliedstaaten voraus. Konsequenz hiervon ist die Delegation der Grenzsicherungsaufgaben an die gemeinsamen Außengrenzen der Europäischen Union (EU). Grenzen in der EU stellen sich somit im Spannungsfeld von Grenzabbau im Innern und massivem Grenzaufbau nach außen dar. Die europasoziologische Grenzforschung fokussiert dieses Spannungsfeld und fragt insbesondere nach den Folgen dieser Politik für die Entwicklung europäischer Gesellschaftsbildung.
\end{abstract}

Schlagwörter

Festung Europa, Binnenfreiheiten, europäische Gesellschaft, Grenzsoziologie

\section{Einleitung: Europäische Grenzzustände soziologisch betrachtet}

Die Schaffung eines gemeinsamen Raums der Freiheit, der Sicherheit und des Rechts sowie die Verwirklichung des freien Binnenmarktes sind für das Europäische Integrationsprojekt konstitutiv. Im Kern allen Bemühens steht der Abbau der Binnengrenzen zwischen den EU-Mitgliedstaaten, um sichtbar die Umsetzung des freien Verkehrs von Kapital, Waren, Dienstleistungen und vor allem Personen zu ermöglichen (die sogenannten vier Binnenmarktfreiheiten). Eben dieses Bemühen der Europäischen Union, sich als einheitlicher Raum zu etablieren, stieß von Anbeginn auf Probleme, hieß dies doch im Umkehrschluss einen deutlichen Verzicht auf Kernaufgaben eines souveränen Staates, eben der Kontrolle des eigenen nationalen Territoriums, zu akzeptieren. Begleitet war dieser Grenzabbau im Innern der EU daher von Beginn an von einem entsprechenden Auf- und Ausbau der Grenzkontrollen an den Außengrenzen der EU (vgl. Eigmüller 2007; Vobruba 2007). Das Thema Grenzen im Kontext der europäischen Integration verortet sich daher von jeher im Spannungsfeld zwischen Grenzabbau einerseits und Grenzausbau andererseits (vgl. Eigmüller 2018).

Der vorliegende Beitrag wird den Stand europasoziologischer Grenzforschung skizzieren und dabei insbesondere seine theoretische Verortung in Georg Simmels Grenzsoziologie darstellen (2), die empirischen Gegebenheiten des europäischen Integrationsprojekts in Hinblick auf Grenzziehung und Grenzöffnung beschreiben (3) und abschließend aktuelle Herausforderungen für eine europasoziologische Sichtweise auf Grenzen diskutieren (4).

\section{Grundlagen europasoziologischer Grenzforschung}

Ausgangspunkt eines europasoziologischen Interesses an Grenzen ist die Tatsache, dass das Europäische Integrationsprojekt zentral von einem gleichzeitigen Abbau der Binnengrenzen im Innern der EU und einem Ausbau der Außengrenzen getragen ist (vgl. Eigmüller 2007; Bach 2010; Eigmüller/Vobruba 2016). Die damit einhergehenden Transformationen von Grenzen, sowohl der Bedeutungsverlust einerseits als auch der immense Bedeutungszuwachs andererseits, sind dabei aber nicht lediglich von politischem oder gar technologischem Interesse, 
sondern vor allem auch von soziologischem Interesse, denn sie zeigen nachhaltig Wirkung auf Gestalt und Wesen der Gesellschaften diesseits und jenseits der Grenze, sowohl in den unmittelbar betroffenen Grenzregionen als auch weit davon entfernt. Hierauf fokussiert die grenzsoziologische Forschung, die die Menschen, die an und mit Grenzen leben, in den Blick nimmt und Prozesse von Grenzaufbau und Grenzabbau im Kontext von Gesellschaftsbildung analysiert (vgl. Eigmüller/Vobruba 2016; siehe auch Schroer in diesem Band). Schon Georg Simmel wies darauf hin, dass nicht die „Grenze eine räumliche Tatsache mit soziologischer Wirkung [ist], sondern eine soziologische Tatsache, die sich räumlich formt" (Simmel 1908/2016, S. 17). Nicht eine Linie macht demnach eine Grenze, sondern vielmehr die Menschen, die an ihr und mit ihr leben und dieser Linie erst die Bedeutung des Ein- beziehungsweise Ausschließens zukommen lassen. Und weiter heißt es:

„Ist sie [die Grenze] freilich erst zu einem räumlich-sinnlichen Gebilde geworden [...], so übt dies starke Rückwirkungen auf das Bewusstsein von dem Verhältnis der Parteien. Während diese Linie nur die Verschiedenheit des Verhältnisses zwischen den Elementen einer Sphäre untereinander und zwischen diesen und den Elementen einer andren markiert, wird sie doch zu einer lebendigen Energie, die jene auseinanderdrängt und sie nicht aus ihrer Einheit herausläßt und sich wie eine physische Gewalt, die nach beiden Seiten hin Repulsion ausstrahlt, zwischen beide schiebt" (Simmel 1908/1992, S. 697).

Simmel betont also die Wirkung der Grenze, die zunächst einmal unabhängig von ihrer physischen Verfasstheit Einfluss auf das Verhalten der Individuen einer Gesellschaft und damit auf die Gestalt der Gesellschaft selbst hat. Entscheidend ist, dass Simmel damit den Blick auf die Frage nach der Wirkung von Grenzen richtet - und nicht auf ihre Entstehung.

In soziologischer Perspektive ist für Simmel die Grenze selbst also nicht bedeutsam, sondern die Grenze wird erst durch die Menschen und deren Beziehungen in Bezug auf die Grenze real (vgl. Eigmüller 2016, S. 70). Einem solchen Verständnis von Grenzen folgend beinhaltet eine empirische Erforschung von Grenzen somit vor allem die Erforschung sozialer Interaktion im Angesicht von Grenzen - und dies umfasst sowohl den Prozess der Grenzkonstitution (bordering) als auch Prozesse des Ausbaus (rebordering) beziehungsweise des Abbaus von Grenzen (debordering; zum Konzept variabler Grenzzustände vgl. insbesondere Houtum/Naerssen 2002; Newman 2011; Sohn 2014).

Damit aber rückt die Grenzwerdung als ein fortwährender sozialer Prozess in den Fokus des Interesses, der sich empirisch beobachten lässt: „Political boundaries are processes and institutions that emerge and exist in boundary-producing practices and discourses, and they may be materialized and symbolized to greater or less extents“ (Paasi 2011, S. 36). Mit Anssi Paasi verstehen wir Grenzen somit als Prozesse, die sich in Praktiken und Diskursen der Grenzziehung materialisieren und Grenzen so erst sichtbar werden lassen (vgl. Lehner in diesem Band; Wille in diesem Band). Dabei gilt, dass Grenzen und ihre Bedeutung nicht auf Dauer gestellt sind, sondern beständigen Transformationen unterliegen. Empirisch eindrucksvoll kann genau dies am Beispiel der europäischen Grenzzustände untersucht werden.

\section{Grenzen in der EU: Inhalte europasoziologischer Grenzforschung}

Das europäische Experiment, so wie es nach dem Ende des 2. Weltkriegs als kühne Vision eines friedlichen und geeinten Kontinents jenseits von Nationalismus und Protektionismus ins Leben gerufen wurde, fußt auf der Idee des Bedeutungsverlustes von Staatsgrenzen. Als Kern 
des europäischen Integrationsprojekts gilt die Verwirklichung der vier Binnenfreiheiten, also des freien Verkehrs von Kapital, Waren, Dienstleistungen und Menschen. Deren Realisierung setzt zugleich den Abbau von Binnengrenzkontrollen zwischen den EU-Mitgliedstaaten voraus. Diese Idee, bereits seit den frühen 1970er-Jahren auf verschiedenen Bühnen immer wieder breit diskutiert, konnte 1986 mit dem intergouvernementalen Vertrag von Schengen für die fünf Kernmitgliedstaaten der damaligen Europäischen Gemeinschaft (EG) - Frankreich, Deutschland, Italien und die Beneluxstaaten - umgesetzt werden. 1995 wurde mit dem Vertrag von Amsterdam diese Abwesenheit von Binnengrenzkontrollen auch in das Gemeinschaftsrecht der EG überführt (vgl. Monar 2000; Eigmüller 2007).

Die Bedeutung des Vertrags von Schengen für das gesamte Integrationsprojekt kann in seiner Wirkung gar nicht hoch genug eingeschätzt werden, denn die an dem Abkommen beteiligten Staaten einigten sich nicht nur auf den Abbau von Binnengrenzkontrollen, also auf den unkontrollierten Austausch von Waren, Kapital und Dienstleistungen, sondern auch darauf, Personen die Grenzen zwischen den Nationalstaaten unkontrolliert passieren zu lassen. Zugleich legte dieses Abkommen auch den Grundstein für ein einheitliches europäisches Grenzregime sowie für einen sich nun entwickelnden gemeinsamen europäischen Rechtsraum (vgl. Eigmüller 2015).

Damit geraten die zwei Achsen der EU-Grenzsicherungspolitik in den Blick: erstens die Bildung eines integrierten Raums „der Freiheit, der Sicherheit und des Rechts“ im Innern, markiert durch die Abwesenheit von Binnengrenzen, und zweitens die zur Herstellung und vor allem Sicherstellung dieses gemeinsamen Raums notwendig gewordene koordinierte Sicherung der Grenzen nach außen - und damit verbunden ein massiver Ausbau der Außengrenzen (vgl. Jureit/Tietze 2015).

\subsection{Grenzausbau an den EU-Außengrenzen}

Bereits im Kontext des Kriegs auf dem Balkan Ende der 1990er-Jahre und spätestens im Zuge der ersten Osterweiterung der Union 2004 kam das Thema des rebordering verstärkt auf die Tagesordnung: nicht nur in der Politik, sondern auch in der sozialwissenschaftlichen Forschung (vgl. Scott/Houtum 2009). Im Unterschied etwa zu politik- oder rechtswissenschaftlichen Studien beschäftigen sich soziologische Analysen des Auf- und Ausbaus der EU-Außengrenzen dabei insbesondere mit Fragen der Wirkung dieses Ausbaus zum einen auf die Anrainerstaaten (vgl. Bigo/Guild 2010), vor allem aber in Hinblick auf die Verfasstheit der EU und ihrer Gesellschaft (vgl. Zielonka 2001; 2003). Grenzsoziologisch bedeutsam waren dabei also weniger Fragen des Integrationsmodus (positive, marktkorrigierende versus negative, marktschaffende Integration; vgl. Scharpf 2008), sondern vielmehr der Prozess der Institutionalisierung der gemeinsamen Grenzsicherungspolitik und damit der Aufbau der Grenze als Institution, die Handlungschancen Einzelner definiert (vgl. Eigmüller 2007; Eigmüller/Vobruba 2016, S. 10). Von Interesse sind dabei sowohl jene staatlichen Akteur*innen, die den Aufbau der EU-Außengrenze vorantreiben bzw. den Diskurs um die Notwendigkeit einer gemeinsamen europäischen Grenzsicherungspolitik bestimmen, als aber auch jene Akteur*innen, die durch ihr auf die Grenze bezogenes Handeln die Grenze selbst in Frage stellen, angreifen und unterwandern. So widmen sich viele Untersuchungen sowohl den Techniken der Grenzsicherung und des Grenzausbaus (Lahav 2004; Bigo/Guild 2005; Walters 2006; Kaufmann 2008) und 
insbesondere der Grenzschutzagentur Frontex (Hess/Kasparek 2010; Müller 2014) als auch der Unterwanderung der Außengrenze v.a. im Kontext von Flucht, Fluchthilfe und Schleusung (z.B. Pfau 2008; Walters 2010; Klepp 2011) sowie Schmuggel (z.B. Bruns 2010; Bruns et al. 2011; allgemein: Horn et al. 2002; Eigmüller 2008).

Insbesondere die politische Soziologie hat sich in einer Reihe von Publikationen zudem mit dem Außenverhältnis des Projekts Europa beschäftigt, das vor allem an der EU-Außengrenze virulent wird. Dabei zielen die Untersuchungen auf die hinter der gemeinsamen Grenzsicherungspolitik liegende Eigenlogik des europäischen Projekts, auf die spezifische „Dynamik Europas“ (Vobruba 2007), die im Fadenkreuz von Integration und Erweiterung ihre Grenzsicherungsfunktionen spezifisch ansiedelt. Ausgehend vom Begriff der Grenze werden die Spannungen zwischen einer entstehenden europäischen Gesellschaft und den politischen Institutionen Europas beschrieben. Die interagierenden Prozesse von Vertiefung und Erweiterung Europas tragen ihre eigene Expansionsdynamik in sich und jede erfolgreich beendete Erweiterungsrunde setzt sowohl Integrations- als auch Exklusionsprozesse in Bezug auf die dann neuen Nachbarn an den neuen Außengrenzen in Gang. Es geht hierbei um die gezielte Einbindung von Anrainerstaaten in das Grenzsicherungsregime der EU, wobei den Anrainerstaaten im Tausch gegen Kooperation im Bereich der Grenzsicherung ein erleichterter Zugang ihrer eigenen Staatsbürger zur EU angeboten wird (vgl. Vobruba 2007; Eigmüller/Vobruba 2010). Ähnliches beschreibt auch Andreas Müller (2014): In seiner Studie am Beispiel der EU-Außengrenze zwischen Polen und der Ukraine zeichnet er detailliert nach, wie sich die EU-Außengrenze hier formiert und zugleich die Ukraine selbst im Rahmen der Europäischen Nachbarschaftspolitik in das europäische Grenzregime inkorporiert wird und mehr und mehr die Grenzsicherungsaufgaben der EU übernimmt. Entscheidende Voraussetzung (und letztlich auch ihre Konsequenz) für diese mutable Grenzsicherungspolitik ist dabei die Variabilität der Gestalt der EU-Außengrenze. Zwar manifestiert sich die EU-Außengrenze ebenso wie auch die Grenzen von Nationalstaaten entlang der Ränder der Europäischen Union, zugleich aber eben auch weit davon entfernt, punktuell im Innern der Union ebenso wie auch in den Anrainerstaaten (vgl. Cuttitta 2015). Man denke nur an die Rechte der Polizei zur verdachtsabhängigen Ausweiskontrolle in den EU-Mitgliedstaaten mit dem Ziel der Feststellung ungültiger Aufenthaltstitel an bestimmten belebten städtischen Plätzen, zu Kontrollen, die der Zoll auf Baustellen mit ebendiesem Ziel durchführt, ebenso wie die Kontrolle von Ausweisen und Visa bereits an Flughäfen im Herkunftsland von Reisenden. Über die Frage von Zugang bzw. Ausschluss zum europäischen Territorium wird somit schon weit vor oder auch erst hinter den Toren der EU entschieden (vgl. Giraudon 2003; Bigo/Guild 2010; Laube 2013). Wir können daher sagen, dass die EU-Außengrenze sowohl eine starre lineare Grenze als auch einen beweglichen Grenzsaum und eine punktuelle Grenze darstellt (vgl. Zielonka 2001; Eigmüller 2007; Cuttitta 2010). Dem teilweisen Bedeutungsverlust herkömmlicher linearer Grenzen steht somit ein Bedeutungszuwachs grenzspezifischer Kontrollfunktionen gegenüber, die aber nicht mehr an den Ort, sondern an das Subjekt geknüpft werden. Die Grenze selbst wird zur „Sortiermaschine“ (Mau 2008), die über Zugang bzw. Ausschluss und damit über Lebenschancen entscheidet.

Insbesondere Jan Zielonka (2003) sowie Didier Bigo und Elsbeth Guild (2010) machen vor dem Hintergrund dieser Entwicklungen immer wieder auch auf die Folgen einer solchen Externalisierung europäischer Grenzsicherungspolitik für die Verfasstheit der EU (insbesondere auf ihre demokratische Verfassung) selbst aufmerksam (vgl. auch Roos 2013). Und hier schließt 
auch das spezifische grenzsoziologische Interesse dieser Befunde an: In grenzsoziologischer Hinsicht interessant sind diese Entwicklungen nicht nur vor der Frage nach der Wirkung dieser gemeinsamen europäischen Außengrenze nach außen. In Anlehnung an Simmel muss eine soziologische Analyse der europäischen Grenzfigurationen insbesondere auch die Wirkung von Grenzabbau wie auch Grenzaufbau nach innen einschließen:

„So ist eine Gesellschaft dadurch, daß ihr Existenzraum von scharf bewußten Grenzen eingefaßt ist, als eine auch innerlich zusammengehörige charakterisiert, und umgekehrt: die wechselwirkende Einheit, die funktionelle Beziehung jedes Elementes zu jedem gewinnt ihren räumlichen Ausdruck in der einrahmenden Grenze. Es gibt vielleicht nichts, was die Kraft insbesondere des staatlichen Zusammenhaltens so stark erweist, als daß diese soziologische Zentripetalität, diese schließlich doch nur seelische Kohärenz von Persönlichkeiten zu einem wie sinnlich empfundenen Bilde einer fest umschließenden Grenzlinie aufwächst“ (Simmel 1908/1992, S. 694).

Dies aber ist eine empirisch noch offene Frage: Führt die Existenz einer gemeinsamen Außengrenze, die eindeutige Feststellung von wir und die anderen, etwa durch entsprechende Unterscheidungen zwischen EU-Bürger*innen und Nicht-EU-Bürger*innen an den Außengrenzposten zur Entstehung und Entwicklung einer gemeinsamen europäischen Identität (vgl. Neumann 1999; Houtum/Naerssen 2002; siehe auch Schwell in diesem Band)? Übernimmt die EU-Außengrenze damit die Funktion, die vormals die nationalstaatlichen Grenzen übernommen haben? Ist sie es nun, die eindeutig Zugehörigkeiten definiert? Die den Zugang zu bestimmten Normen und Rechten garantiert, die nur im Innern, aber nicht außen gelten? Markiert sie einen bestimmten Rechtsraum, einen Raum bestimmter politischer, rechtlicher und sozialer Ordnung? Und welche sozialen Stratifikationen gehen mit diesen neuen Grenzziehungen einher? Welche neuen sozialen Ungleichheiten werden durch sie markiert?

\subsection{Europäische Prozesse des Grenzabbaus}

Während die Außengrenzen der EU politisch also deutlich aufgewertet und damit in Folge als Grenzen umso wahrnehmbarer wurden, wurden die Binnengrenzen (zumindest eine gewisse Zeit lang) politisch nahezu bedeutungslos.

Soziologisch betrachtet schloss sich hier zunächst die Frage an, inwieweit insbesondere Menschen, die an diesen Grenzen leben, diesen in ihren alltäglichen Grenzpraktiken entsprechend den politischen Vorstellungen tatsächlich zu Bedeutungslosigkeit bzw. Bedeutungszuwachs verhalfen und wie sich dieser Bedeutungswandel von Grenzen konkret gestaltet (vgl. v.a. Deger/Hettlage 2007; Eder 2007; Roose 2010). Dabei zeigen diverse grenzsoziologische Studien sehr eindrücklich, dass die Frage der Bedeutung politischer Grenzen immer eine ist, die in einem Wechselspiel zwischen einerseits politischer und rechtlicher Setzung und andererseits gesellschaftlicher Akzeptanz und Umsetzung bzw. Subversion und Angriff ausgehandelt wird (vgl. Horn et al. 2002; Eigmüller 2008).

Von besonderem Interesse für die europasoziologische Forschung sind dabei grenzsoziologische Studien, die entlang der Grenzbildungsprozesse des Auf- bzw. Abbaus von Grenzen Identitätskonstruktionen diesseits und jenseits von Grenzen untersuchen. $\mathrm{Zu}$ nennen sind hier etwa Studien, die in den Euroregionen entlang der EU-Binnengrenzen nach Identitätskonstruktionen, Wandel von Zugehörigkeiten und der Herausbildung neuer (kultureller und sozialer) 
Grenzen fragen (vgl. Immerfall 2000; Delhey 2004; Roose 2010; Opiłowska 2011; Gasparini 2014). Jüngere Studien nehmen zudem Bezug auf die aktuellen Entwicklungen im europäischen Grenzregime und fragen nach den gesellschaftlichen Folgen - insbesondere in Hinblick auf eine europäische Gesellschaftsbildung - angesichts eines zunehmenden Wiederaufbaus von Binnengrenzen (vgl. Scuzzarello/Kinnvall 2013; Börzel/Risse 2018).

\section{Ausblick: Herausforderungen grenzsoziologischer EU-Forschung}

Europasoziologische Grenzforschung fokussiert im Kontext von Grenzbildung somit auf zweierlei: Zum einen auf die Prozesse der Abschottung an den EU-Außengrenzen sowie auf die Folgen dieser Abschottungspolitik in Hinblick auf die Konstitution europäischer Gesellschaft. Fragen, die sich hieran anschließen, umfassen damit sowohl die Entstehung und Entwicklung eines europäischen Selbstverständnisses als auch die Folgen dieser Abschottungspolitik für die hiervon betroffenen Gesellschaften außerhalb der EU. Zum anderen nimmt eine Soziologie der EU-Grenzen die Folgen des Grenzabbaus im Innern der Union in den Blick und fragt nach diesen Prozessen des debordering insbesondere in den hiervon betroffenen Regionen entlang der Binnengrenzen der Union. Wie nachhaltig sind diese Prozesse des Grenzabbaus? Wo entstehen zugleich neue Grenzen oder manifestieren sich alte Grenzen auf neue Weise? Welchen Einfluss hat der politische Grenzabbau auf die Entwicklung von Grenzidentitäten?

Wie der kursorische Blick auf die europäischen Grenzzustände gezeigt hat, sind es insbesondere zwei Fragen, die auch in der europasoziologischen Grenzforschung seit Beginn der 2000erJahre vermehrt verhandelt werden: die Frage der Wirkung von Grenzen nach innen, auf das Selbstverständnis und die Identität von Menschen im Innern eines von Grenzen umschlossenen Raums sowie die Frage der Wirkung von Grenzen nach außen, nach ihrer Wirkmacht, individuelle Lebenschancen zu gestalten. Grundlegend an diesen grenzsoziologischen Europastudien war dabei von Beginn an, dass nicht nur dem Raum hinter bzw. vor der Grenze Beachtung geschenkt wurde, sondern immer mehr die Grenze selbst zum Gegenstand des Interesses wurde (vgl. Eigmüller/Vobruba 2016; siehe auch Gerst/Krämer in diesem Band). Damit hat das vielfältige Forschungsfeld der Grenzsoziologie auch in der soziologischen Europaforschung seinen festen Platz eingenommen.

Beide Forschungsfelder, sowohl die soziologische Grenzforschung als auch die soziologische Europaforschung, haben dabei vom Blick auf den jeweils anderen Forschungsgegenstand profitiert: Die soziologische Europaforschung konnte mit dem Rückgriff auf grenzsoziologische Theorien die Bedingungen, unter denen Gesellschaftsbildung in der EU vonstatten geht und von denen sie maßgeblich determiniert ist, genauer erfassen und exakter interpretieren (vgl. Gerst/Krämer 2017; Schiffauer et al. 2018). Die soziologische Grenzforschung hingegen konnte mit dem Fokus auf die Europäische Integration ein reichhaltiges empirisches Feld für sich nutzbar machen, in dem Prozesse des Grenzabbaus mit denen des Grenzaufbaus einhergingen, und vor dem Hintergrund vielfältiger historischer Entwicklungen Fragen zur Veränderung von Grenzkonstellationen und Gesellschaftsbildung untersuchen (vgl. Eigmüller 2007; Laube 2013; Hilpert 2020).

Die vielen vorliegenden Studien belegen dabei eindrücklich, dass in Folge der europäischen Integration nicht nur ein Grenzabbau im Innern der EU stattgefunden hat, sondern es an den Binnengrenzen vor allem zu einer Veränderung von Grenzgegebenheiten gekommen ist. Im Gegensatz zum Nationalstaat werden Grenzen innerhalb der EU nun nicht mehr in erster Linie 
durch Staaten und deren Politik gebildet. Vielmehr finden Prozesse des bordering in der EU nun weit jenseits davon statt. Während staatliche Grenzen abgebaut werden, gewinnen die Prozesse des alltäglichen bordering dabei zunehmend an Bedeutung (vgl. Rumford 2007).

Was aber bedeutet es für die europäische Gesellschaft, dass die Binnengrenzen - mal mehr, mal weniger sichtbar - zumindest als kulturelle Grenzen, als Sprachgrenzen und auch als politische Grenzen - weiter existieren und sogar noch an Bedeutung gewinnen? Gerade die Entwicklungen im Rahmen der seit 2015 virulenten sogenannten ,Flüchtlingskrise“ haben dabei einmal mehr die gemeinsame EU-Grenzsicherungspolitik in Frage gestellt und die Brüchigkeit sowohl des Abkommens von Schengen (1986) als auch der Abkommen mit Drittstaaten zu deren Integration ins Schengen-System verdeutlicht. Und die Leichtigkeit, mit der die Binnengrenzen jederzeit wieder aktiviert werden können und freies Reisen und grenzüberschreitendes Leben im europäischen Raum jäh an den wiedererrichteten Binnengrenzen stoppt, zeigte sich im Frühjahr 2020 im Zuge der sogenannten ,Corona-Epidemie‘ mit ungeahnter Deutlichkeit. Ob dies langsam, aber sicher das Ende des europäischen Projekts einläutet oder ob die EU sich von diesen Wochen nationalstaatlicher Renaissance im Krisenmodus erholen wird, ist derzeit noch offen. Allerdings zeigen gerade diese jüngsten Entwicklungen einmal mehr die zentrale Bedeutung, die territorialen Grenzen (sowohl Staats- als auch Landesgrenzen) im kollektiven Bewusstsein und insbesondere als politisches Steuerungsinstrument und zugleich als Gegenstand von Symbolpolitik zukommt. Fest steht, dass der enorme Forschungsbedarf im Feld der europasoziologischen Grenzforschung auch weiterhin gegeben ist. Insbesondere Fragen nach der politischen Verfasstheit der EU im Kontext dieser spezifischen europäischen Grenzzustände stellen sich nun verschärft, und zwar nicht nur in Bezug auf die Wiedererrichtung der Binnengrenzen, sondern auch insbesondere in Bezug auf den weiteren massiven Ausbau ihrer Außengrenzen.

Angesichts andauernder Menschenrechtsverletzungen im Kontext der EU-Grenzsicherungspolitik (vgl. Cuttitta 2014; 2018; Carrera/Hertog 2015; Rijpma/Vermeulen 2015) muss über Wert und Inhalt von Demokratie und Rechtsstaatlichkeit in Europa neu nachgedacht werden und zugleich gefragt werden, wie Gesellschaftsbildung unter diesen Voraussetzungen aussehen kann: Was heißt es für die europäische Gesellschaft, wenn an ihren Außengrenzen täglich Menschen bei dem Versuch sterben, die Grenze zu überwinden, um Teil des europäischen Projektes zu werden (vgl. auch Banse in diesem Band)? Und was bedeutet es für die europäische Gesellschaft, wenn ihr Kernanliegen, das ,grenzenlose Europa', zum Spielball nationalistischer Interessenpolitik wird? Was bedeutet es für die europäischen Gesellschaften, wenn diese aufgrund der Grenzzustände und entlang der Frage ihrer Bearbeitung zunehmend gespalten sind? Welche Werte setzen sich langfristig auf Ebene der Bevölkerungen durch? Welche Zukunft hat der Versuch, grenzüberschreitend solidarisches Verhalten zu etablieren und Netzwerke zu fördern, wenn entlang der Grenzfrage Abgrenzung, Protektionismus und Nationalismen die Politik bestimmen? Welche Antwort kann eine europasoziologische Forschung, die ausgehend von Grenzen Gesellschaftsbildung analysiert, auf den deutlichen Zuspruch für Populisten und antieuropäische Kräfte, die das politische Geschehen innerhalb der EU zunehmend beeinflussen, heute geben? Da die gegenwärtig gesellschaftlich und politisch relevanten Fragen vor allem solche sind, die sich nur jenseits von Nationalstaaten und über Staatsgrenzen hinweg sinnvoll bearbeiten lassen, spricht auch zukünftig vieles dafür, dass die Grenzen auch weiterhin durchlässig bleiben. Zugleich aber sind es diese offenen Nationalstaatsgrenzen und die für die EU spezifischen dynamischen Grenzzustände sowie die damit einhergehenden variablen 
Mitgliedschaftsräume (vgl. Bös 2000), die immer offener von nationalistischen und antieuropäischen Kräften in vielen EU-Mitgliedstaaten angegriffen und in Frage gestellt werden.

Gerade diese jüngsten Konflikte zeigen dabei einmal mehr, welches Potential für die soziologische Europaforschung in einem grenzsoziologischen Zugang steckt und welche Bedeutung dieser Forschung zukünftig noch zukommen wird. Vor allem jüngere Ansätze, die neue Methoden und Theorien grenzsoziologischer Forschung in den Mittelpunkt rücken - wie etwa Ansätze der Narratologie oder auch der Arbeit mit visuellen Daten (z.B. Christmann 2014; 2016; Banks/Zeitlyn 2015) -, lassen hier auf wesentliche Erkenntnisse auch für die zukünftige grenzsoziologische Europaforschung hoffen.

\section{Weiterführende Literatur}

Christmann, Gabriela (Hrsg.) (2016): Zur kommunikativen Konstruktion von Räumen. Wiesbaden: VS Verlag für Sozialwissenschaften.

Eigmüller, Monika/Vobruba, Georg (Hrsg.) (2016): Grenzsoziologie. Die politische Strukturierung des Raums. 2., überarb. Aufl., Wiesbaden: VS Verlag für Sozialwissenschaften.

Jureit, Ulrike/Tietze, Nikola (2015): Postsouveräne Territorialität. Eine Einleitung. In: Dies. (Hrsg.): Postsouveräne Territorialität. Die Europäische Union und ihr Raum. Hamburg: Hamburger Edition, S. 724.

Vobruba, Georg (2007): Die Dynamik Europas. 2. akt. Aufl. Wiesbaden: VS Verlag für Sozialwissenschaften.

\section{Literaturverzeichnis}

Bach, Maurizio (2010): Die Konstitution von Räumen und Grenzbildung in Europa. Von verhandlungsresistenten zu verhandlungsabhängigen Grenzen. In: Eigmüller, Monika/Mau, Steffen (Hrsg.): Gesellschaftstheorie und Europapolitik. Wiesbaden: VS Verlag für Sozialwissenschaften, S. 153-178.

Banks, Marcus/Zeitlyn, David (2015): Visual Methods in Social Research. London: Sage.

Bigo, Didier/Guild, Elsbeth (2005): Policing at a distance: Schengen visa policies. In: dies. (Hrsg.): Controlling Frontiers: Free movement into and within Europe. Houndmills: Routledge, S. 233-263

Bigo, Didier/ Guild, Elsbeth (2010): The Transformation of European Border Controls. In: Ryan, Bernard/Mitsilegas, Valsamis (Hrsg.): Extraterritorial Immigration Control. Legal Challenges. Leiden: Brill, S. 252-273.

Börzel, Tanja/Risse, Thomas (2018): From the euro to the Schengen crises: European integration theories, politicization, and identity politics. In: Journal of European Public Policy 25, H. 1, S. 83-108.

Bös, Mathias (2000): Zur Kongruenz sozialer Grenzen. Das Spannungsfeld von Territorien, Bevölkerungen und Kulturen in Europa. In: Bach, Maurizio (Hrsg.): Die Europäisierung nationaler Gesellschaften. Kölner Zeitschrift für Soziologie und Sozialpsychologie, Sonderheft 40, Wiesbaden: Westdeutscher Verlag, S. 429-455.

Bruns, Bettina (2010): Grenze als Ressource: Die soziale Organisation von Schmuggel am Rande der Europäischen Union. Wiesbaden, VS Verlag für Sozialwissenschaften.

Bruns, Bettina/Miggelbrink, Judith/Müller, Kristine (2011): Smuggling and small-scale trade as part of informal economic practices. In: International journal of sociology and social policy 31, H. 11/12, S. 664-680.

Carrera, Sergio/Hertog, Leonhard den (2015): Whose Mare? Rule of Law Challenges in the Field of European Border Surveillance in the Mediterranean. In: CEPS Paper in Liberty and Security 79, 2015.

Christmann, Gabriela (2014): Investigating Spatial Transformation Processes. An Ethnographic Discourse Analysis in Disadvantaged Neighbourhoods. In: Historical Social Research 39, H. 2, S. 235-256.

Christmann, Gabriela (Hrsg.) (2016): Zur kommunikativen Konstruktion von Räumen. Wiesbaden: VS Verlag für Sozialwissenschaften.

Cuttitta, Paolo (2010): Das europäische Grenzregime: Dynamiken und Wechselwirkungen. In: Hess, Sabine/Kasparek, Bernd (Hrsg.): Grenzregime. Diskurse, Praktiken, Institutionen in Europa. Berlin: Assoziation-A, S. 23-40.

Cuttitta, Paolo (2014): From the Cap Anamur to Mare Nostrum: Humanitarianism and Migration Controls at the EU's Maritime Borders. In: CLEER Working Papers 7, 2014.

Cuttitta, Paolo (2015): Territorial and Non-territorial: the Mobile Borders of Migration Controls. In: Amilhat Szary, Anne-Laure/Giraut, Frédéric (Hrsg.): Borderities and the Politics of Contemporary Mobile Borders. Houndmills: Routledge, S. 241-255. 
Cuttitta, Paolo (2018): Delocalization, humanitarianism, and human rights: The Mediterranean border between exclusion and inclusion. In: Antipode 50, H. 3, S. 783-803.

Deger, Petra/Hettlage, Robert (Hrsg.) (2007): Der europäische Raum. Die Konstruktion europäischer Grenzen. Wiesbaden: VS Verlag für Sozialwissenschaften.

Delhey, Jan (2004): European social integration: from convergence of countries to transnational relations between peoples. In: WZB Discussion Paper 201, 2004.

Eder, Klaus (2007): Die Grenzen Europas. Zur narrativen Konstruktion europäischer Identität. In: Deger, Petra/Hettlage, Robert (Hrsg.): Der europäische Raum. die Konstruktion europäischer Grenzen. Wiesbaden: VS Verlag für Sozialwissenschaften, S. 187-208.

Eigmüller, Monika (2007): Grenzsicherungspolitik. Funktion und Wirkung der europäischen Außengrenze. Wiesbaden: VS Verlag für Sozialwissenschaften.

Eigmüller, Monika (2008): Subversionen an Staatsgrenzen. In: Comparativ 18, H. 1, S. 13-22.

Eigmüller, Monika (2015): Die Entwicklung des europäischen Rechtsraums als sozialpolitischer Anspruchsraum: Raumdimensionen der EU-Sozialpolitik. In: Jureit, Ulrike/Tietze, Nikola (Hrsg.): Postsouveräne Territorialität. Die Europäische Union und ihr Raum. Hamburg: Hamburger Edition, S. 255272.

Eigmüller, Monika (2016): Der duale Charakter der Grenze. Bedingungen einer aktuellen Grenztheorie. In: Dies./Vobruba, Georg (Hrsg.): Grenzsoziologie. Die politische Strukturierung des Raums. 2., überarb. Aufl., Wiesbaden: VS Verlag für Sozialwissenschaften, S. 55-73.

Eigmüller, Monika (2018): Grenzsicherungspolitik, europäische. In: Bach, Maurizio/Hönig, Barbara (Hrsg.): Europasoziologie. Handbuch für Wissenschaft und Studium. Baden-Baden: Nomos, S. 180185.

Eigmüller, Monika/Vobruba, Georg (2010): Selektive Grenzöffnung im Rahmen der Europäischen Nachbarschaftspolitik. In: Möllers, Martin H. W./Ooyen, Robert Chr. van (Hrsg.): Migration, Integration und europäische Grenzpolitik. Frankfurt/M: Verlag für Polizeiwissenschaft, S. 493-503.

Eigmüller, Monika/Vobruba, Georg (Hrsg.) (2016): Grenzsoziologie. Die politische Strukturierung des Raums. 2., überarb. Aufl., Wiesbaden: VS Verlag für Sozialwissenschaften.

Gasparini, Alberto (2014): Belonging and identity in the European border towns: Self-centered borders, hetero-centered borders. In: Journal of Borderlands Studies 29, H. 2, S. 165-201.

Gerst, Dominik/Krämer, Hannes (2017): Methodologische Prinzipien einer allgemeinen Grenzsoziologie. In: Lessenich, Stephan (Hrsg.): Geschlossene Gesellschaften. Verhandlungen des 38. Kongresses der Deutschen Gesellschaft für Soziologie. Bamberg, 2016.

Giraudon, Virginie (2003): The constitution of a European immigration policy domain: a political sociology approach. In: Journal of European public policy 10, H. 2, S. $263-282$.

Hess, Sabine/Kasparek, Bernd (2010): Grenzregime. Diskurse, Praktiken, Institutionen in Europa. Berlin: Assoziation-A.

Hilpert, Isabel (2020): Die doppelt codierte Grenze und der Nationalstaat in Europa. Wiesbaden: VS Verlag für Sozialwissenschaften.

Horn, Eva/Kaufmann, Stefan/Bröckling, Ulrich (2002): Grenzverletzer: Von Schmugglern, Spionen und anderen subversiven Gestalten. Berlin: Kadmos.

Houtum, Henk van/Naerssen, Ton van (2002): Bordering, ordering and othering. In: Tijdschrift voor Economische en Sociale Geografie 93, H. 2, S. 125-136.

Immerfall, Stefan (2000): Fragestellungen einer Soziologie der europäischen Integration. In: Bach, Maurizio (Hrsg.): Die Europäisierung nationaler Gesellschaften. Wiesbaden: Westdeutscher Verlag, S. 481503.

Jureit, Ulrike/Tietze, Nikola (2015): Postsouveräne Territorialität. Eine Einleitung. In: Dies. (Hrsg.): Postsouveräne Territorialität. Die Europäische Union und ihr Raum. Hamburg: Hamburger Edition, S. 724.

Kaufmann, Stefan (2008): Technik als Politik. In: Comparativ 18, H. 1, S. $42-57$.

Klepp, Silja (2011): Europa zwischen Grenzkontrolle und Flüchtlingsschutz: Eine Ethnographie der Seegrenze auf dem Mittelmeer. Bielefeld: transcript.

Lahav, Gallya (2004): Immigration and politics in the new Europe: Reinventing borders. Cambridge: Cambridge University Press.

Laube, Lena (2013): Grenzkontrollen jenseits nationaler Territorien: Die Steuerung globaler Mobilität durch liberale Staaten. Frankfurt/M: Campus.

Mau, Steffen/Laube, Lena/Roos, Christof/Wrobel, Sonja (2008): Grenzen in der globalisierten Welt. Selektivität, Internationalisierung, Exterritorialisierung. In: Leviathan. Berliner Zeitschrift für Sozialwissenschaft 36 , H. 1, S. 123-148. 
Monar, Jörg (2000): Die Entwicklung des „Raumes der Freiheit, der Sicherheit und des Rechts “: Perspektiven nach dem Vertrag von Amsterdam und dem Europäischen Rat von Tampere. In: Integration 23, H. 1, S. 18-33.

Müller, Andreas (2014): Governing mobility beyond the state: Centre, periphery and the EU's external borders. Basingstoke: Palgrave Macmillian.

Neumann, Iver B. (Hrsg.) (1999): Uses of the other. „The East“ in European identity formation. Manchester: Manchester University Press.

Newman, David (2011): Contemporary research agendas in border studies: An overview. In: Wastl-Walter, Doris (Hrsg.): The Ashgate Research Companion to Border Studies. Farnham: Ashgate, S. 55-70.

Opiłowska, Elżbieta (2011): Stadt - Fluss - Grenze: Geteilte Städte an der deutsch-polnischen Grenze. In: Eurostudia 7, H. 1-2, S. $153-166$.

Paasi, Anssi (2011): A Border Theory: An Unattainable Dream or a Realstic Aim for Border Scholars? In: Wastl-Walter, Doris (Hrsg.): The Ashgate Research Companion to Border Studies, Farnham: Ashgate, S. 33-54.

Pfau, Jonas (2008): Prekäre Migration und Ausschluss. In: Comparativ 18, H. 1, S. $23-41$.

Rijpma, Jorrit/Vermeulen, Mathias (2015): EUROSUR: Saving Lives or Building Borders? In: European Security 24, H. 3, S. 454-472.

Roos, Christof (2013): EU and Immigration Policies: Cracks in the Walls of Fortress Europe? Basingstoke: Palgrave Macmillan.

Roose, Jochen (2010): Vergesellschaftung an Europas Binnengrenzen. Wiesbaden: VS-Verlag.

Rumford, Chris (2007): Does Europe have Cosmopolitan Borders? In: Globalizations 4, H. 3, S. 327-339.

Scharpf, Fritz W. (2008): Negative und positive Integration. In: Die politische Ökonomie der europäischen Integration. Frankfurt/M: Campus, S. 49-87.

Schiffauer, Werner/Koch, Jochen/Reckwitz, Andreas/Schoor, Kerstin/Krämer, Hannes (2018): Borders in Motion. Durabilität, Permeabilität, Liminalität. In: Working Paper Series B/Orders in Motion. DOI:10.11584/B-ORDERS. 1

Scott, James W./Houtum, Henk van (2009): Reflections on EU territoriality and the „bordering“ of Europe. In: Political Geography 5, H. 28, S. 271-273.

Scuzzarello, Sarah/Kinnvall, Catarina (2013): Rebordering France and Denmark narratives and practices of border-construction in two European countries. In: Mobilities 8, H. 1, S. 90-106.

Simmel, Georg (1908/1992): Soziologie. Untersuchungen über die Formen der Vergesellschaftung. Kapitel IX. In: Ders.: Georg Simmel Gesamtausgabe. Herausgegeben von Otthein Rammstedt. Frankfurt/M: Suhrkamp, S. 687-698.

Simmel, Georg (1908/2016): Der Raum und die räumlichen Ordnungen der Gesellschaft. In: Eigmüller, Monika/Vobruba, Georg (Hrsg.): Grenzsoziologie. Die politische Strukturierung des Raums. 2. überarb. Aufl., Wiesbaden: VS Verlag für Sozialwissenschaften, S. 9-17.

Sohn, Christophe (2014): Modelling cross-border integration: The role of borders as a resource. In: Geopolitics 19, H. 3, S. 587-608.

Vobruba, Georg (2007): Die Dynamik Europas. 2. akt. Aufl. Wiesbaden: VS Verlag für Sozialwissenschaften.

Walters, William (2006): Border/control. In: European journal of social theory 9, H. 2, S. 187-203.

Walters, William (2010): Deportation, Expulsion, and the International Police of Aliens. In: Genova, Nicholas de/Mae Peutz, Nathalie/Walters, William/Cornelisse, Galina (Hrsg.): The Deportation Regime: Sovereignty, Space, and the Freedom of Movement. Durham: Duke University Press, S. 69-100.

Zielonka, John (2001): How new enlarged borders will reshape the European Union. In: Journal of Common Market Studies 39, H. 3, S. 507-536.

Zielonka, John (Hrsg.) (2003): Europe unbound: Enlarging and reshaping the boundaries of the European Union. Houndmills: Routledge. 